\title{
Prevención de la transmisión materno-infantil del virus de inmunodeficiencia humana de tipo 1 en Uruguay: 1987-2009. Análisis de las diferentes medidas adoptadas
}

\author{
Jorge Quian R., Stella Gutiérrez R., Virginia González C., Ana Visconti G., \\ Gabriela Sicco C., Cecilia Murialdo E. y Marta Lioni H.
}

\section{Prevention of vertical transmission of the human immunodeficiency virus type 1 in Uruguay: 1987-2009. An analysis of measures adopted}

The prevention of vertical transmission (VT) of human immunodeficiency virus (HIV) is a priority as it generally is an avoidable disease. Aim: To describe Uruguay's experience of VT during 23 years and the preventive measures adopted. Patients and Methods: Children of HIV positive mothers born between January 1987 and December 2009 were followed. The mother, pregnancy and children dependent variables were analysed. Infants were classified as infected, not infected, exposed but still in evaluation, or withdrawn of the protocol. The different measures implemented were analyzed. Results: There were 1353 children of HIV positive mothers; 204 (15.1\%) infants were infected. The percentage of VT diminished throughout the years; between 2003 and 2009 it decreased to $6 \%$. Protective factors against HIV VT were antiretroviral therapy (ARV) during pregnancy, elective caesarean section and not to nurse the newborn infant. Women who did not receive ARV had a percentage of VT of $50.3 \%$. Conclusion: The VT has diminished in Uruguay. Special efforts must be done to screen all pregnant women, to improve diagnosis during pregnancy and to implement suitable ARV as occurs in developed countries.

Key words: HIV, vertical transmission, prevention.

Palabras clave: VIH, transmisión vertical, prevención.

\section{Introducción}

$\mathrm{E}$ n el año 2008 se estimaba que había 33,4 millones de personas infectadas por el virus de inmunodeficiencia humana (VIH), de los cuales 15,7 millones eran mujeres y 2,1 millones tenían menos de 15 años de edad habiendo sido infectados primariamente a través de la transmisión materno infantil (TMI ${ }^{1-5}$. Al momento actual, la infección de niños por esta vía es en gran medida evitable por lo que la prevención de la transmisión vertical del VIH es una prioridad mundial.

En los primeros años de la pandemia, la sangre y sus productos derivados constituyeron otra fuente de transmisión, pero el desarrollo de los estudios de pesquisa y los controles sobre los donantes de sangre han hecho esta vía excepcional ${ }^{6,7}$.

En el Centro Hospitalario Pereira Rossell (CHPR) funciona desde 1990 el Centro Nacional de Referencia Obstétrico Pediátrico VIH-SIDA. En el mismo, en sus inicios solamente se realizaba el control de los niños hijos de madre con infección por VIH; desde 1998 se realiza también el control de las mujeres embarazadas infectadas. Luego del nacimiento, si el niño resulta infectado, se realiza la totalidad del seguimiento y se ofrece tratamiento al mismo; en caso contrario se deriva al primer nivel de atención para que continúe con los controles pediátricos de rutina.

Uruguay tiene aproximadamente 3.200 .000 habitantes y las distancias territoriales mayores no exceden los 600 kilómetros. En el momento actual este Centro asiste prácticamente a $90 \%$ de los hijos de madres seropositivas para VIH del país.

En épocas pasadas, cuando aún no se realizaba intervención alguna para evitar la transmisión vertical, la tasa de transmisión para países europeos oscilaba entre 15 y $25 \%$ y entre 25 y $45 \%$ para países en desarrollo ${ }^{8}$. En Uruguay esta tasa llegó a ser de $50 \%$.

A lo largo del tiempo, diversas medidas han sido implementadas con el fin de lograr el control de la TMI. La primera fue la supresión de la lactancia materna ${ }^{10}$. Actualmente algunas investigaciones han demostrado que junto al uso materno de anti-retrovirales (ARV) el amamantamiento sería posible. Desde un enfoque de riesgo, en países donde la morbi-mortalidad infantil en ausencia de lecha materna es elevada, es aconsejable preservar la lactancia natural ${ }^{11,12}$.

En 1994 Connors y cols., demostraron que el tratamiento con zidovudina (ZDV) a la mujer durante el
Centro Hospitalario Pereira Rossell. Montevideo Uruguay. Centro VIH-SIDA Obstétrico Pediátrico.

No hubo conflictos de interés No existieron fuentes de financiación.

Recibido: 28 de marzo de 2011 Aceptado: 11 de octubre de 2011

Correspondencia a: Jorge Quian Rivero jorgeq@internet.com.uy 
embarazo, trabajo de parto y al recién nacido, disminuía la TMI, llegando a cifras de $8 \%{ }^{13}$. Esta pauta también demostró buenos resultados en otros países ${ }^{14}$.

En los años siguientes, con biterapia, en general ZDV y lamivudina (3TC), las cifras de transmisión continuaron descendiendo ${ }^{15,16}$ y finalmente se recomendó el tratamiento de alta eficacia (TAE) con tres ARV para lograr cargas virales $(\mathrm{CV})$ indetectables con lo que la transmisión llegó a cifras de 1 a $2 \%{ }^{17,18}$.

La cesárea electiva, antes del inicio del trabajo de parto y de la ruptura de membranas demostró también descenso de la TMI ${ }^{19}$. Pero si la mujer embarazada tiene una CV $<1.000$ copias/ml, la vía de nacimiento no parece influir de manera decisiva en la $\mathrm{TMI}^{20}$.

Uruguay tiene una prevalencia de VIH en la población general de $0,41 \%$ y desde 1997 , está pautado que el equipo de salud debe ofrecer el test de VIH a la mujer embarazada. Dado que un porcentaje importante de mujeres del sector público del país no controlan su embarazo o lo hacen de forma incompleta ${ }^{21}$ desde 2001 se comenzó a realizar el test rápido de $\mathrm{VIH}^{22}$ a las mujeres que ingresaban para tener a su hijo sin controles previos.

La principal maternidad pública está ubicada en el Hospital de la Mujer del CHPR y asiste un total de 8.000 a 9.000 nacimientos por año (18\% del total del país).

La mayoría de las evidencias referidas a TMI proceden de países de altos o de muy bajos ingresos. Uruguay está calificado por el Banco Mundial ${ }^{23}$ como un país de ingresos medio-alto, por lo que se considera esta experiencia puede ser de utilidad para otros países de la región.

El objetivo de esta comunicación fue presentar la evolución de las cifras de TMI del VIH en el país a lo largo de 23 años de trabajo. Como objetivos secundarios describir las distintas acciones adoptadas a lo largo del tiempo para prevenir la TMI y evaluar sus resultados.

\begin{tabular}{|c|c|}
\hline Año & Acciones implementadas \\
\hline 1990 & $\begin{array}{l}\text { Supresión de la lactancia materna } \\
\text { Entrega de leche modificada hasta los } 6 \text { meses de vida }\end{array}$ \\
\hline 1996 & ARV durante el embarazo, parto y al recién nacido \\
\hline 1997 & $\begin{array}{l}\text { Inclusión del test de VIH en el control de la mujer embarazada } \\
\text { Posibilidad de medir carga viral } \\
\text { Terapia doble ARV }\end{array}$ \\
\hline 1998 & $\begin{array}{l}\text { Terapia triple ARV } \\
\text { Control de la mujer embarazada infectada en el Centro VIH-SIDA }\end{array}$ \\
\hline 2000 & Cesárea electiva, lo que luego se modificó de acuerdo a la carga viral \\
\hline 2002 & Test rápido de VIH. De ser positivo se confirma con ELISA y Western-Blot \\
\hline $2008-2009$ & Test de resistencia a los ARV \\
\hline
\end{tabular}

\section{Material y Métodos}

Se realizó un estudio descriptivo, retrospectivo.

\section{Población del estudio}

Se incluyeron hijos de madre seropositivas para VIH nacidos entre el $1^{\circ}$ de enero de 1987 y el 31 de diciembre de 2009 y controlados en el Centro Nacional de Referencia Obstétrico-Pediátrico VIH-SIDA del CHPR.

La fuente de información fueron las historias clínicas de los niños asistidos en el Centro. Estas son historias codificadas, completadas por ginecólogos y pediatras tratantes durante la asistencia y volcadas posteriormente a un programa de computación por uno de los autores (JQ).

Las variables estudiadas fueron: fecha de nacimiento, edad de la madre, número de embarazo, ARV recibidos durante el embarazo y el parto, ARV recibidos por el RN, edad gestacional, modo de nacer (cesárea de elección, que es la planificada junto a la madre y realizada antes del inicio del trabajo de parto, cesárea de urgencia, parto vaginal), sexo, peso al nacer, amamantamiento y duración del mismo y clasificación del niño.

Definiciones. Se consideró infectado (I) a los lactantes bajo 18 meses de edad, con dos RPC positivas o una RPC positiva y signos clínicos de enfermedad y en los niños sobre 18 meses de edad, con ELISA y WesternBlot confirmatorio; no infectado o serorevertido (S) a los efectos de la presente comunicación a los que tenían tres RPC negativas, una después del $4^{\circ}$ mes de nacer o ELISA negativo luego de los 18 meses y expuestos sin clasificar (E) a los que abandonaron los controles sin haber completado los exámenes correspondientes o al momento del estudio no tenían edad suficiente para tener todos los exámenes necesarios.

En muchos casos no se pudo obtener todos los datos debido a abandono familiar, fallecimiento de la madre, institucionalización o adopción.

Se excluyó a los niños infectados por vía transfusional o por abuso sexual.

La Tabla 1 describe las distintas acciones implementadas a lo largo del tiempo para prevenir la TMI del VIH.

A los efectos de la presente evaluación, se dividió los 23 años de trabajo en cuatro períodos según la presencia de diferentes medidas preventivas: previo a 1990; 19911996; 1997-2002 y 2003-2009.

Los resultados de la prevención de la TMI fueron analizados según el año de nacimiento del niño e incluidos en el período correspondiente.

\section{Análisis estadístico}

Se calcularon porcentajes con sus correspondientes intervalos de confianza de 95\%. Se utilizó el test de $\chi^{2}$ y se consideró significativa una $\mathrm{p}<0,05$. Se utilizó el programa Epi-Info versión 6.04 


\section{Resultados}

Entre el $1^{\circ}$ de enero de 1987 y el 31 de diciembre de 2009 se registraron 1.353 hijos de madre infectada por el VIH.

Un niño fue excluido del análisis ya que concurrió a una sola consulta y no se obtuvo la fecha de nacimiento.

Quedaron para el análisis 1.352 niños cuyos nacimientos de acuerdo a los grupos de años fueron: 12 antes de 1990; 222 entre 1991-1996; 492 entre 1997 y 2002 y 626 entre 2003 y 2009.

Hubo 13 parejas de gemelares; 1 de los 26 resultó infectado, fue el primer gemelar de una madre que no recibió ARV y que tuvo un parto vaginal. Dos permanecieron como expuestos. Se contabilizaron como casos individuales.

La distribución por sexo mostró 691 (51,1\%) masculinos y $661(48,9 \%)$ femeninos.

Del total, 204 resultaron infectados $(15,1 \%$ IC $95 \%$ $13,2-17,1), 1.107(81,8 \%)$ fueron no infectados y 41 (3\%) permanecieron como expuestos sin clasificar.

La Tabla 2 describe la evolución de las cifras de TMI del VIH en los niños incluidos durante 23 años, en relación con las principales medidas preventivas adoptadas.

Se contó con el dato del peso al nacer en 1.312 registros $(97 \%) ; 1.089(83 \%)$ pesaron igual o más de 2.500 gramos y entre ellos $158(14,5 \%)$ resultaron infectados; $223(16,9 \%)$ pesaron menos de 2.500 gramos y entre ellos $20(8,9 \%)$ resultaron infectados $(\mathrm{p}<0,05)$.

La Tabla 3 describe las características de los grupos de niños (de acuerdo al peso mayor o menor de 2.500 gramos) en cuanto a ARV durante el embarazo, alimentación con pecho materno, vía de nacimiento y en qué período, de acuerdo al año, nacieron.

Se encontraron diferencias estadísticamente significativas en relación a la cesárea de urgencia y en la cantidad de nacimientos en el período 2003-2009; en las restantes variables las diferencias no fueron significativas.

Se conoce la edad gestacional de 1.257 (92,9\%) niños;
$1.079(85,8 \%)$ fueron recién nacidos de término $(\geq 37$ semanas) y de ellos 140 (13\%) fueron infectados. 178 niños fueron de pre-término, de ellos $20(11,2 \%)$ fueron infectados $(\mathrm{p}>0,05)$.

Se contó con el dato sobre lactancia materna en 1.291 $(95 \%)$ registros; $1.139(88 \%)$ no fueron amamantados y

Tabla 2. Evolución de las cifras de TMI del VIH en los niños incluidos durante los 23 años en relación con las principales medidas preventivas adoptadas

\begin{tabular}{|c|c|c|c|c|c|}
\hline Año & $\begin{array}{c}\mathrm{n} \text { de } \\
\text { nacimientos }\end{array}$ & $\begin{array}{c}\text { Infectados } \\
\text { n (\%) }\end{array}$ & $\begin{array}{c}\text { Expuestos } \\
\text { sin clasificar } \\
\text { "n" }\end{array}$ & $\begin{array}{c}\text { Acciones } \\
\text { adoptadas en } \\
\text { el tiempo }\end{array}$ & $\begin{array}{l}\text { TMI en los } \\
\text { distintos } \\
\text { períodos }\end{array}$ \\
\hline 1987 & 2 & $2(100)$ & 0 & & \multirow{4}{*}{$67 \%$} \\
\hline 1988 & 2 & $2(100)$ & 0 & & \\
\hline 1989 & 2 & $1 \quad(50)$ & 0 & & \\
\hline 1990 & 6 & $3 \quad(50)$ & 0 & No lactancia & \\
\hline 1991 & 22 & $10 \quad(45)$ & 0 & & \multirow{6}{*}{$36 \%$} \\
\hline 1992 & 20 & $8 \quad(40)$ & 0 & & \\
\hline 1993 & 33 & $12 \quad(36)$ & 3 & & \\
\hline 1994 & 45 & $12 \quad(26)$ & 3 & & \\
\hline 1995 & 38 & $15 \quad(39)$ & 0 & & \\
\hline 1996 & 64 & $24 \quad(37)$ & 5 & ARV progresivos & \\
\hline 1997 & 54 & $12 \quad(22)$ & 1 & Test embarazo & \multirow{6}{*}{$16 \%$} \\
\hline 1998 & 66 & $10 \quad(15)$ & 1 & & \\
\hline 1999 & 74 & $10 \quad(13)$ & 1 & & \\
\hline 2000 & 91 & $17 \quad(18)$ & 2 & Cesárea.electiva & \\
\hline 2001 & 103 & $20 \quad(19)$ & 2 & & \\
\hline 2002 & 104 & $10 \quad(9)$ & 0 & Test rápido & \\
\hline 2003 & 98 & (8) & 3 & & \multirow{7}{*}{$6 \%$} \\
\hline 2004 & 98 & (8) & 2 & & \\
\hline 2005 & 109 & (8) & 2 & & \\
\hline 2006 & 74 & $3 \quad(4)$ & 1 & & \\
\hline 2007 & 89 & (2) & 6 & & \\
\hline 2008 & 86 & $3 \quad(3)$ & 4 & & \\
\hline 2009 & 72 & $3 \quad(4)$ & 5 & & \\
\hline Total & 1.352 & $204 \quad(15)$ & 41 & & \\
\hline
\end{tabular}

TMI: transmisión materno-infantil.

\section{Tabla 3. Características de los niños menores e iguales o mayores a 2.500 grs. según tratamiento ARV materno durante el embarazo,} lactancia materna, vía y año de nacimiento

\begin{tabular}{|c|c|c|c|c|c|c|c|c|c|}
\hline \multirow[b]{2}{*}{ Peso } & \multirow[b]{2}{*}{$\begin{array}{c}\text { ARV en } \\
\text { embarazo }\end{array}$} & \multirow[b]{2}{*}{ LM } & \multicolumn{3}{|c|}{ Vía de nacimiento } & \multicolumn{4}{|c|}{ Períodos anuales } \\
\hline & & & $\begin{array}{l}\text { Cesárea } \\
\text { electiva }\end{array}$ & $\begin{array}{c}\text { Cesárea } \\
\text { urgencia }\end{array}$ & $\begin{array}{c}\text { Parto } \\
\text { vaginal }\end{array}$ & 1987-1990 & 1991-1996 & 1997-2002 & 2003-2009 \\
\hline $\begin{array}{l}<2.500 \text { grs } \\
\text { N: } 223(16,9 \%)\end{array}$ & $\begin{array}{c}137 \\
(61,4 \%)\end{array}$ & $\begin{array}{c}13 \\
(5,8 \%)\end{array}$ & $\begin{array}{c}78 \\
(35 \%)\end{array}$ & $\begin{array}{c}63 \\
(28 \%)\end{array}$ & $\begin{array}{c}80 \\
(35.8 \%)\end{array}$ & 1 & $\begin{array}{c}24 \\
(10,7 \%)\end{array}$ & $\begin{array}{c}69 \\
(30,9 \%)\end{array}$ & $\begin{array}{c}129 \\
(57,8 \%)\end{array}$ \\
\hline \multirow[t]{2}{*}{$\begin{array}{l}\geq 2.500 \text { grs } \\
N: 1.089(83 \%)\end{array}$} & $\begin{array}{c}674 \\
(61,9 \%)\end{array}$ & $\begin{array}{c}132 \\
(12 \%)\end{array}$ & $\begin{array}{c}473 \\
(43,4 \%)\end{array}$ & $\begin{array}{c}98 \\
(8,9 \%)\end{array}$ & $\begin{array}{c}507 \\
(46,5 \%)\end{array}$ & $\begin{array}{c}6 \\
(0,5 \%)\end{array}$ & $\begin{array}{c}183 \\
(16,8 \%)\end{array}$ & $\begin{array}{c}412 \\
(37,8 \%)\end{array}$ & $\begin{array}{c}488 \\
(44,8 \%)\end{array}$ \\
\hline & $p>0,05$ & $p>0,05$ & $p>0,05$ & $p<0,05$ & $p>0,05$ & & $p>0,05$ & $p>0,05$ & $p<0,05$ \\
\hline
\end{tabular}


de estos se infectaron 82 (7,2\%); fueron amamantados 152 niños y $96(63,2 \%)$ se infectaron $(\mathrm{p}<0,05)$.

La edad de la madre constaba en 1.330 casos (98\%); $1.167(87,5 \%)$ tenían 20 o más años. De ellas $166(14 \%)$ fueron niños infectados; 163 madres tenían menos de 20 años de edad y tuvieron 26 niños infectados $(15,9 \%)$ $(\mathrm{p}>0,05)$.

La vía de nacimiento se obtuvo en 1.311 (97\%) registros; 595 (45,4\%) nacieron por parto vaginal, 554 $(42,3 \%)$ por cesárea programada y $162(12,4 \%)$ por cesárea de urgencia. De acuerdo a la forma de nacer resultaron infectados: por parto vaginal 137 (23\%), por cesárea programada $19(3,4 \%)$ y por cesárea de urgencia $24(14,8 \%)$. La diferencia entre los tres grupos fue significativa $(\mathrm{p}<0,05)$.

En cuanto a los ARV recibidos durante el embarazo para evitar la TMI, 813 mujeres recibieron ARV, 508 no los recibieron (132 de ellas antes de 1995) y en 31 no fue posible obtener datos. De las 813 que recibieron ARV, 328 recibieron monoterapia con AZT, 311 recibieron triple terapia y 168 recibieron doble terapia. Hubo 3 mujeres que recibieron $\mathrm{AZT}$ y se desconoce si recibieron otro ARV; hubo dos mujeres que recibieron doble terapia pero no fue posible saber si recibieron o no otro ARV. Una mujer recibió monoterapia con nevirapina.

La Tabla 4 describe las características de la población estudiada en relación con los modos de nacer, la medicación recibida y la lactancia materna.

Entre las 508 que no recibieron ARV durante el embarazo, 395 tampoco los recibieron durante el parto vaginal o la cesárea. De esos recién nacidos, 302 no recibieron ARV y resultaron infectados $152(50,3 \%)$ y permanecieron como expuestos sin clasificar $11(3,6 \%)$; 93 recién nacidos recibieron ZDV y se infectaron nueve $(9,3 \%)$ (Figura 1).

Las restantes 104 mujeres que no recibieron ARV durante el embarazo, sí los recibieron en el momento del parto vaginal o la cesárea; 103 recién nacidos también recibieron ARV y se infectaron $16(15,5 \%)$; uno no recibió y resultó no infectado (Figura 1).

\section{Tabla 4. Número de nacimientos, vías de nacimiento, sin tratamiento con ARV, monoterapia con ZDV durante el embarazo, bi-terapia y} tri-terapia y quienes recibieron pecho materno de acuerdo al año de nacimiento

\begin{tabular}{|c|c|c|c|c|c|c|c|c|c|c|}
\hline \multirow[b]{2}{*}{ Año } & \multirow[b]{2}{*}{$\begin{array}{c}\mathrm{n} \text { de } \\
\text { nacimientos }\end{array}$} & \multirow[b]{2}{*}{$\begin{array}{l}\text { Cesárea } \\
\text { electiva }\end{array}$} & \multicolumn{2}{|c|}{ Modo de nacer } & \multicolumn{6}{|c|}{ Medicación para TMI } \\
\hline & & & $\begin{array}{c}\text { Cesárea de } \\
\text { urgencia }\end{array}$ & $\begin{array}{c}\text { Parto } \\
\text { vaginal }\end{array}$ & Sin datos & Sin ARV & ZDV & $\begin{array}{c}\mathrm{Bi}- \\
\text { terapia }\end{array}$ & $\begin{array}{c}\text { Tri- } \\
\text { terapia }\end{array}$ & $\begin{array}{c}\text { Lactancia } \\
\text { materna }\end{array}$ \\
\hline 1987 & 2 & 0 & 0 & 2 & 0 & 2 & 0 & 0 & 0 & 2 \\
\hline 1988 & 2 & 0 & 0 & 1 & 1 & 2 & 0 & 0 & 0 & 0 \\
\hline 1989 & 2 & 0 & 0 & 1 & 1 & 2 & 0 & 0 & 0 & 1 \\
\hline 1990 & 6 & 1 & 0 & 4 & 1 & 6 & 0 & 0 & 0 & 1 \\
\hline 1991 & 22 & 3 & 1 & 16 & 2 & 22 & 0 & 0 & 0 & 6 \\
\hline 1992 & 20 & 2 & 1 & 15 & 2 & 20 & 0 & 0 & 0 & 5 \\
\hline 1993 & 33 & 8 & 3 & 19 & 3 & 33 & 0 & 0 & 0 & 10 \\
\hline 1994 & 45 & 6 & 2 & 33 & 4 & 43 & 1 & 0 & 0 & 12 \\
\hline 1995 & 38 & 5 & 2 & 28 & 3 & 33 & 5 & 0 & 0 & 8 \\
\hline 1996 & 64 & 5 & 5 & 50 & 4 & 43 & 20 & 0 & 0 & 13 \\
\hline 1997 & 54 & 7 & 3 & 44 & 0 & 23 & 25 & 5 & 0 & 11 \\
\hline 1998 & 66 & 7 & 8 & 49 & 2 & 20 & 35 & 8 & 2 & 11 \\
\hline 1999 & 74 & 19 & 15 & 39 & 1 & 20 & 36 & 13 & 4 & 6 \\
\hline 2000 & 91 & 39 & 8 & 43 & 1 & 29 & 38 & 14 & 9 & 14 \\
\hline 2001 & 103 & 48 & 20 & 32 & 3 & 34 & 37 & 22 & 8 & 16 \\
\hline 2002 & 104 & 60 & 13 & 30 & 1 & 29 & 48 & 21 & 6 & 6 \\
\hline 2003 & 98 & 58 & 8 & 30 & 2 & 25 & 40 & 15 & 13 & 7 \\
\hline 2004 & 98 & 62 & 10 & 23 & 3 & 24 & 21 & 31 & 19 & 5 \\
\hline 2005 & 109 & 60 & 20 & 27 & 2 & 29 & 9 & 19 & 50 & 6 \\
\hline 2006 & 74 & 38 & 11 & 24 & 1 & 16 & 5 & 7 & 44 & 2 \\
\hline 2007 & 89 & 38 & 11 & 38 & 2 & 18 & 2 & 9 & 55 & 4 \\
\hline 2008 & 86 & 50 & 9 & 25 & 2 & 22 & 3 & 2 & 54 & 5 \\
\hline 2009 & 72 & 38 & 12 & 22 & 0 & 13 & 3 & 2 & 47 & 1 \\
\hline Total & 1.352 & 554 & 162 & 595 & 41 & 508 & 328 & 168 & 311 & 152 \\
\hline
\end{tabular}


En nueve casos no se obtuvieron datos de medicación en parto y recién nacido.

Recibieron AZT como único ARV 328 mujeres; 14 niños resultaron infectados $(4,3 \%$ IC $95 \% 2,-7,2)$ y 5 permanecieron como expuestos.

De las 168 mujeres que recibieron doble terapia ARV (la gran mayoría con ZDV y 3TC; todas las combinaciones incluyeron ZDV), ZDV durante el nacimiento y los recién nacidos ZDV por 42 días, no amamantados, dos niños se infectaron (1,2\%; IC 95\% 0,2 - 4,8) (uno nació por cesárea de urgencia y no recibió la madre ZDV intravenosa y el otro por cesárea electiva y se cumplió con todo el protocolo) y tres permanecieron como expuestos $(1,8 \%)$. El modo de nacer fue en 96 casos (58\%) por cesárea de elección, en 42 casos (26\%) por vía vaginal y en los 25 restantes por cesárea de urgencia. En un caso no se tuvieron datos.

De las 311 mujeres que recibieron triple terapia (siempre con ZDV salvo un caso) y los recién nacidos ZDV por 42 días y no amamantados, cuatro niños se infectaron (1,3\%; IC95\% 0,4-3,5). Tres cumplieron con todo el protocolo y uno nació por cesárea de urgencia. Permanecieron como expuestos 10 niños (3,2\%). El modo de nacer fue en 204 casos por cesárea electiva, en 63 por parto vaginal, en 42 por cesárea de urgencia y en dos casos se desconoce el dato.

El porcentaje de cesáreas electivas fue similar en los grupos que recibieron doble o triple terapia ARV $(\mathrm{p}>0,05)$.

El número de mujeres que recibió doble o triple terapia, las vías de nacimiento, la ausencia de ARV en el embarazo, la monoterapia con ZDV, la bi y tri-terapia y quienes recibieron pecho materno, se presentan en la Tabla 3.

\section{Discusión}

El presente estudio abarca 23 años de análisis de la TMI del VIH en el Uruguay. En ese tiempo hubo múltiples variaciones en los enfoques terapéuticos que han repercutido positivamente sobre dicha transmisión. Cada vez menos niños son infectados por el VIH si se toman las medidas adecuadas durante el embarazo. El porcentaje global de TMI en los 23 años fue de 15,1\%. Esta cifra es muy elevada. El análisis por períodos demuestra un descenso desde $67 \%$ en el primero a $6 \%$ en el último. Si bien las cifras han descendido, ya desde el año 1998 y siguientes se publicaban resultados de TMI de entre $1 \mathrm{y}$ $2 \%$ en los países desarrollados ${ }^{19-24,25}$.

Es necesario reducir aún más este porcentaje. El país cuenta con todos los elementos para lograrlo: hay acceso a los equipos de salud, los ARV son gratuitos, es posible suministrar suplementos lácteos a los niños. Pero el eslabón inicial de un sistema de salud adecuado no se ha logrado para parte de la población: el control adecuado del

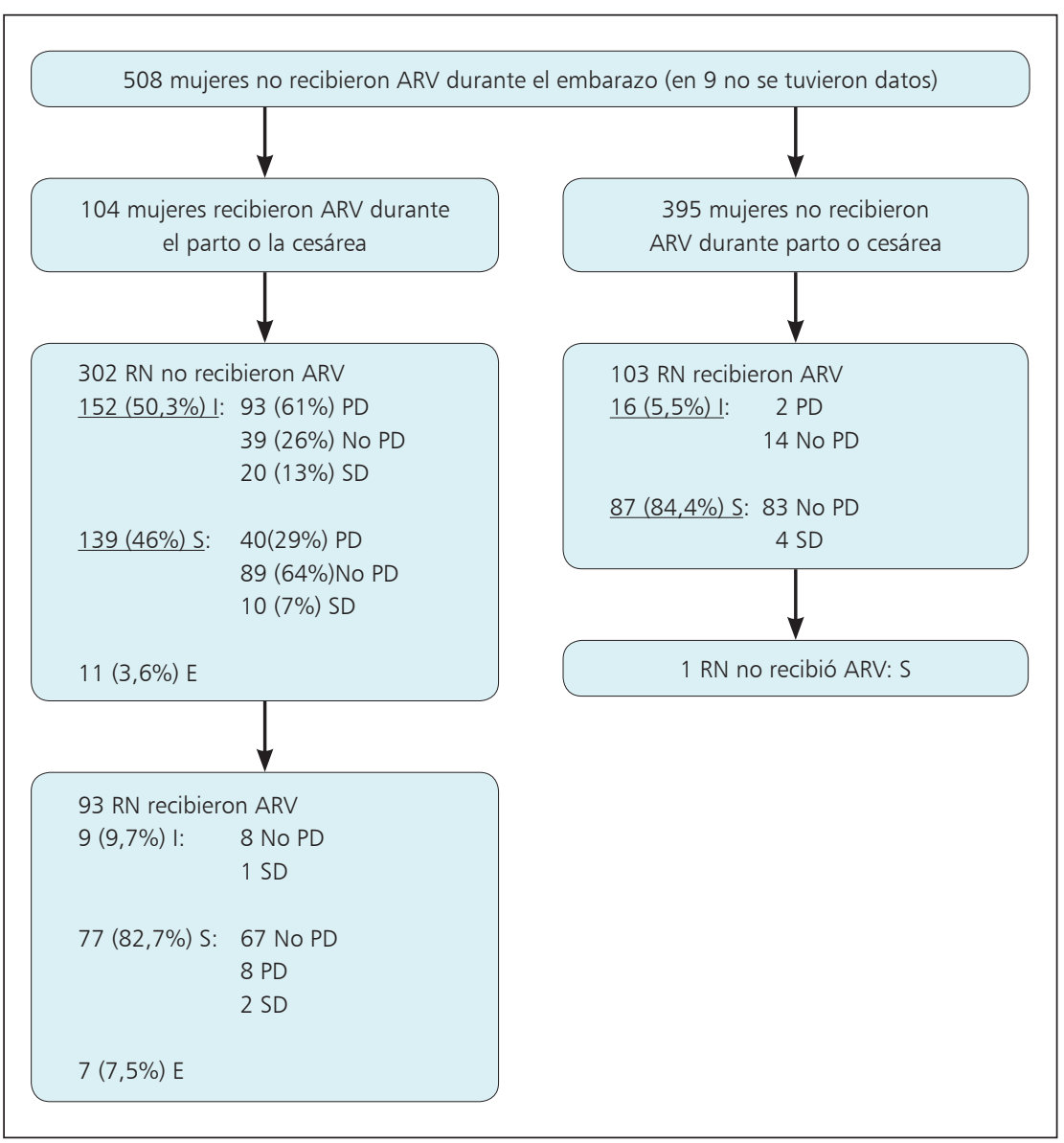

Figura 1. Evolución de los recién nacidos de mujeres que no recibieron ARV durante el embarazo, de acuerdo a si recibieron o no ARV en el parto o el recién nacido y relación con el amamantamiento. $\mathrm{PD}$ = pecho directo; $\mathrm{SD}$ = sin datos; I: infectados; S: serorevertidos; E: expuestos (indefinidos).

embarazo, el cual disminuye la TMI del VIH pero también la disminución o desaparición de otras enfermedades de transmisión vertical que influyen en la morbi-mortalidad de los niños.

Los obstáculos más importantes para la erradicación de la TMI en Uruguay son el aumento de la infección VIH en mujeres en edad fértil, la ausencia o retardo del cuidado prenatal, sobre todo en mujeres que consumen sustancias ilícitas, la infección primaria aguda en etapas tardías del embarazo o en quienes están amamantando, la mala adherencia a los regímenes de ARVs prescritos y la falta de implementación rutinaria y en tiempos reales del test de VIH con la imprescindible consejería ${ }^{26}$.

Son necesarias campañas para lograr que todas las mujeres controlen su embarazo desde el inicio como lo hacen países que en el momento actual se plantean la posibilidad de erradicar el SIDA pediátrico ${ }^{21,27}$.

Por otra parte, los equipos de salud deben estar atentos 
cuando llega a la maternidad una mujer que no ha tenido controles adecuados. Se ha demostrado que incluso la utilización de ARV en el momento del parto, el administrar ARV al recién nacido y evitar la lactancia contribuyen a disminuir la $\mathrm{TMI}^{28,29}$.

En el presente estudio las mujeres que no reciben ARV en momento alguno del embarazo ni del parto y tampoco el recién nacido, tienen porcentaje de transmisión de $50,3 \%$; cuando los reciben durante el parto ese porcentaje disminuye francamente. Cuando se administra ARV al recién nacido y a su vez se indica evitar la lactancia los porcentajes de transmisión descienden en forma significativa.

No se encontraron diferencias en la TMI en relación con el sexo del niño, como encuentran algunos estudios que sugieren que las niñas estarían más propensas a infectarse "in utero" que los varones ${ }^{30}$.

Tampoco hubo diferencias en cuanto a la posibilidad de infectarse en relación a la edad gestacional del niño ni a la edad de la madre.

El peso al nacer mostró una mayor asociación de TMI en los que pesaron más de 2.500 gramos que en los más pequeños. La mayoría de estudios muestran datos opuestos, donde el bajo peso se asocia con mayor frecuencia de infección ${ }^{31}$.

De los datos que pudieron ser analizados, solamente se encontraron diferencias significativas estadísticamente en cuanto a cesárea de urgencia (fue mayor en los neonatos bajo 2.500 gramos) y en el período de nacimiento (un porcentaje mayor de los niños bajo 2.500 gramos nacieron entre 2003-2009). No parece que estos datos tengan importancia significativa. No se investigaron otros factores contundentes como la carga viral, el consumo de sustancias ilícitas y otras enfermedades de transmisión sexual que pudieron influir en los resultados.

El análisis de las vías de nacimiento no es posible hacerlo sin tener en cuenta que las mujeres que tuvieron cesárea electiva probablemente controlaron mejor su embarazo y recibieron ARV durante el mismo. Sin embargo, la cesárea demostró su efecto protector no sólo en su uso programado sino también en la de urgencia al compararla con la TMI registrada con parto vaginal ${ }^{19,24}$.

El elemento más significativo fue el hallazgo de resultados similares de TMI entre quienes recibieron doble y triple terapia, si bien el intervalo de confianza es mayor para el primer grupo. Ambos grupos fueron además comparables en cuanto al porcentaje de cesáreas y a que en ambos, ninguno de los niños fue amamantado.

Townsend y cols., analizando la TMI demuestran que utilizando solamente ZDV en madres que no requieren tratamiento (con determinación de $\mathrm{CV}$ ) y que terminan en operación cesárea prácticamente no tienen infección en los niños ${ }^{32}$. Esto es de suma importancia y se deberán replantear las políticas de profilaxis en las mujeres que no necesitan tratamiento por su enfermedad y que se comprometen con el cuidado de su embarazo, que no usan drogas tanto las llamadas sociales (alcohol, tabaco) como las ilícitas. Exponer a menor cantidad de ARV al feto es importante y además desde el punto de vista económico significa un ahorro importante para la sociedad, que como la de nuestro país, ofrece gratuitamente los ARV a la población.

También se considera de importancia que quienes reciben ARV ya sea como profilaxis de la TMI o como por tratamiento, no amamanten a sus hijos.

Está demostrado que en los países donde el reemplazo de la leche materna es realizable, accesible, aceptable, sustentable en el tiempo y seguro, se debe continuar con la política sanitaria de no amamantar, incluso en las mujeres que reciben ARV en el post-parto. Uruguay puede y debe mantener esta política.

El presente estudio tiene varias debilidades. En primer término el plazo extendido de 23 años muestra muy diferentes forma de la prevención de la TMI. Esto sucedió en todo el mundo y por tanto resulta difícil la comparación de los distintos resultados. Demuestra además la dificultad que tienen algunos países en tener a disposición medicamentos de importancia. En efecto, Uruguay tuvo acceso limitado a ZDV a partir de 1996 y el estudio de Connors ${ }^{13}$ ya se había publicado en 1994 con lo cual se perdieron años de oportunidades.

Tampoco se tomaron en cuenta algunos elementos que son de valor para la evaluación de la TMI como el nivel de CD4 maternos y la CV. En general, es difícil contar con estos valores en tiempo real (demoran a veces casi un mes en obtener sus resultados). Hay estudios que demuestran que la CV es importante como datos de la globalidad de la población pero que para el caso individual su valor se ve reducido ya que se ha visto TMI del VIH incluso con CV indetectables.

En Uruguay se redujo lentamente la TMI a cifras que todavía no son adecuadas. El 6\% registrado en el último período con la disponibilidad de todos los recursos descritos es producto de embarazos aún mal controlados ya que las cifras de transmisión en mujeres con bi o triterapia son muy bajas.

\section{Resumen}

Introducción: La prevención de la transmisión materno infantil (TMI) del VIH es una prioridad debido a que está demostrado que es una infección prácticamente evitable. Objetivo: Presentar cifras de TMI durante 23 años junto a las distintas medidas adoptadas. Pacientes y Métodos: Hijos de madre con infección por VIH nacidos entre el 1/I/1987 y el 31/XII/2009. Se estudiaron variables con respecto a la madre, el embarazo y el niño. Se clasificaron a los niños en infectados, no infectados y expuestos sin 
clasificar por estudio pendiente o abandono del protocolo. Se analizaron las acciones implementadas. Resultados: Hubo 1.353 hijos de madre infectadas por VIH; se excluyó 1. Resultaron 204 infectados (15,1\%). El porcentaje de TMI disminuyó a lo largo de los años; entre 2003 y 2009 y en el último período llegó a $6 \%$. Fueron factores protectores para disminuir la TMI el haber recibido terapia anti- retroviral (TARV) durante el embarazo, no amamantar y la cesárea electiva. Las mujeres que no recibieron TARV tuvieron un porcentaje de TMI de 50,3\%: Discusión: La TMI ha disminuido en Uruguay. Se deben realizar esfuerzos para captar la mujer embarazada, diagnosticarla y ofrecer TARV adecuado para lograr cifras similares a la de países desarrollados.

\section{Referencias}

1.- Joint United Nations Programme on HIV/AIDS and World Health Organization. AIDS epidemic update, December 2009. Geneva, Switzerland. http://data.unaids.org/pub/Report/2009/2009_ epidemic_update_en.pdf (accedido $10 \mathrm{de}$ noviembre 2010).

2.- Blanche S, Rouzioux C, Guihard M, Veber F, Mayaux M, Jacomet C, et al. Prospective study of infants born to women seropositive for HIV type 1. N Engl J Med 1989; 320: 1643-8.

3.- Nduati R, Grace J, Mbori-Ngacha D, Richardson B, Overbaugh J, Mwatha A, et al. Effect of breastfeeding and formula feeding on transmission of HIV-1: a randomized clinical trial. JAMA 2000; 283 (9): 1167-74.

4.- Arbona S, Melville S, Hanson C, Squuires J, Doyle M, Doran T, et al. Mother-to-child transmission of the HIV in Texas. Pediatr Infect Dis J. 2001; 20: 602-6.

5.- Fiscus S, Adimora A, Funk M, Schoenbach V, Tristram D, Lim W, et al. Trends in interventions to reduce perinatal HIV type 1 transmission in North Carolina. Pediatr Infect Dis J. 2002; 21: 664-8.

6.- Centers for Disease Control and Prevention. Provisional public health service inter-agency. Recommendations for screening donated blood and plasma for antibody to the virus causing acquired immunodeficiency syndrome. MMWR Morb Mortal Wkly Rep 1985; 34: 1-5.

7.- Quinn T, Riggin C, Kline R, Francis H, Mulanga K,Sension M, Fauci A. Rapid latex agglutination assay using recombinant envelope polypetide for the detection of antibody to the HIV. JAMA, 1988; 260: 510-3.

8.- Thorne C, Newell M. Mother to child transmission of HIV and its prevention. Curr HIV Res 2003; 4: 447-62.

9.- Quian J, Gutiérrez S, Picón T, Visconti A, González A, Nin M, et al. Transmisión vertical del VIH según quimioprofilaxis recibida, vía de nacimiento y amamantamiento. Rev Med Urug 2002; 18: 265-9.
10.- Ruff A, Halsey N, Coberly J. Breast-feeding and maternal-infant transmission of HIV type 1. J Pediatr 1992; 121 (2): 325-9.

11.- Shapiro R, Hughes M, Ogwu A, Kitch D, Lockman S, Moffat C, et al. Antiretroviral regimens in pregnancy and breast-feeding in Botswana. N Engl J Med 2010; 362: 2282-94.

12.- Peltier C, Ndayisaba G, Lepage P, van Griensven J, Leroy V, Pharm C, et al. Breastfeeding with maternal antiretroviral therapy or formula feeding to prevent HIV postnatal mother-to-child transmission in Rwanda. AIDS 2009; 23: 2415-23.

13.- Connor E M, Sperling R, Gelfer R, Kiselev P, Scott G.,O Sullivan MJ. Reduction of maternalinfant transmission of HIV-type 1 with zidovudine treatment. N Engl J Med 1994; 331: 1173-80.

14.- Quian J, Picón T, Rodríguez I, Gutiérrez S, Gonsalez A, Nin M, et al. Evaluación de la quimioprofilaxis con zidovudina a la mujer embarazada VIH (+) y su hijo. Arch Pediatr Urug 2001; 72(1): 72-6.

15.- Mandelbrot L, Landreau-Mascaro A, Rekacewicz C, Berrebi A, Bénifla J, Burgard M, et al. Lamivudine-zidovudine combination for prevention of maternal-infant transmission of HIV-1. JAMA 2001; 285: 2083-93.

16.- McGowan J, Crane M, Wiznia A, Blum S. Combination antiretroviral therapy in HIVinfected pregnant women. Obstet Gyenecol 1999; 94: 641-6.

17.- European Collaborative Study. HIV-infected pregnant women and vertical transmission in Europe since 1986. AIDS 2001; 15: 761-70.

18.- Ioannidis J, Abrams E, Ammann A, Bulterys M, Goedert J, Gray L, et al. Perinatal transmission of HIV type 1 by pregnant women with RNA virus loads $<1.000$ copies $/ \mathrm{ml}$. J Infect Dis 2001; 183: 539-45.

19.- The European Mode of Delivery Collaboration. Elective caesarean-section versus vaginal delivery in prevention of vertical HIV-1 transmission: a randomised clinical trial. Lancet 1999; 353: 1035-9.
20.- European Collaborative Study. Mother-to-child transmission of HIV infection in the era of highly active antiretroviral therapy. Clin Infect Dis 2005; 40; 458-65.

21.- Quian J, Gutiérrez S, Zabala C, González V, Bernadá E, Güimil S, et al. Oportunidades perdidas para evitar la transmisión maternoinfantil del VIH; Uruguay 2005-2007. Rev Med Urug 2009; 25: 27-33.

22.- Quian J, Visconti A, Gutiérrez S, Galli A, Maturo M, Galeano V, et al. Detección de infección por VIH a través de test rápido en mujeres embarazadas; una estrategia exitosa para disminuir su transmisión vertical. Rev Chil Infectol 2005; 22 (4): 321-6.

23.- http://siteresources worldbank.org/ DATASTATISCS/Resources; acceso el 9/X/2010.

24.- Kind C, Rudin C, Siegrist C, Wyler C, Biedermann K, Lauper U et al. Prevention of vertical HIV transmission: additive protective effect of elective caesarean section and zidovudine prophylaxis. AIDS 1998; 12: 205-10.

25.- Mandelbrot L, Le Chenadec J, Berrebi A, Bongain A, Benfila J, Delfraissy J, et al. Perinatal HIV-1 transmission: interaction between zidovudine prophylaxis and mode of delivery in the French perinatal cohort. JAMA 1998; 280: 55-60.

26.- Panel on treatment of HIV pregnant women and prevention of perinatal transmission. Recommendations for use of antiretroviral drugs in pregnant HIV-1-infected women for maternal health and interventions to reduce perinatal HIV transmission in the United States. May 24; 2010; pp 1-117. Available at http://aidsinfo.nih. gov/ContentFiles/PerinatalGL.pdf (accedido 16 de noviembre 2010).

27.- Rogers M, Taylor A, Nesheim S. Preventing perinatal transmission of HIV: the national perspective. J Public Health Manag Pract 2010, 16 (6): 505-8.

28.- Taha T, Kumwenda N, Gibbons A, Broadhead R, Fiscus S, Lema V, et al. Short 
post exposure prophylaxis in newborn babies to reduce mother-to-child transmission of HIV-1; NVAZ randomised clinical trial. Lancet 2003; 362: 1171-7.

29.- Gray G, Urban M, Chersich M, Bolton C, van Niekerk R, Violary A, et al. A randomized trial of two post-exposure prophylactic regimens to reduce mother-to-child HIV-1 transmission in infants of untreated mothers. AIDS 2005; 19 : 1289-97.

30.- European Collaborative Study. Are girls more at risk of intrauterine-acquired HIV infection than boys? AIDS 2004; 18 (2): 344-7.

31.- Townsend C, Cortina-Borja M, Peckham S, Tookey P. Antiretroviral therapy and premature delivery in diagnosed HIV-infected women in the United Kingdom and Ireland. AIDS 2007; 21: 1019-26.

32.- Townsend C, Cortina-Borja M, Peckham C, de Ruiter A, Lyall H, Tookey P. Low rates of mother-to-child transmission of HIV following effective pregnancy interventions in the United Kingdom and Ireland, 2000-2006. AIDS 2008; 22: $973-81$. 